\title{
Optimal timing for antihypertensive dosing: focus on valsartan
}

\author{
Ramón C Hermida' \\ Diana E Ayala' \\ Carlos Calvo²
}

'Bioengineering \& Chronobiology Laboratories, University of Vigo,

Campus Universitario, Vigo, 36200

Spain; ${ }^{2}$ Hypertension and Vascular

Risk Unit, Hospital Clinico

Universitario, Santiago de

Compostela, 15706 Spain
Correspondence: Ramón C Hermida

Director, Bioengineering and

Chronobiology Labs, E.T.S.I.

Telecomunicación, Campus Universitario,

VIGO (Pontevedra) 36200, Spain

Tel: 34-986-8| 2 | 48 \& 34-986-8| 2146

Fax: 34-986-812116

Email:rhermida@uvigo.es

\begin{abstract}
Some specific features of the $24 \mathrm{~h}$ blood pressure (BP) pattern are linked to the progressive injury of target tissues and the triggering of cardiac and cerebrovascular events. In particular, many studies show the extent of the nocturnal BP decline relative to the diurnal BP mean (the diurnal/nocturnal ratio, an index of BP dipping) is deterministic of cardiovascular injury and risk. Normalization of the circadian BP pattern is considered to be an important clinical goal of pharmacotherapy because it may slow the advance of renal injury and avert end-stage renal failure. The chronotherapy of hypertension takes into account the epidemiology of the BP pattern, plus potential administration-time determinants of the pharmacokinetics and dynamics of antihypertensive medications, as a means of enhancing beneficial outcomes and/or attenuating or averting adverse effects. Thus, bedtime dosing with nifedipine gastrointestinal therapeutic system (GITS) is more effective than morning dosing, while also reducing significantly secondary effects. The dose-response curve, therapeutic coverage, and efficacy of doxazosin GITS are all markedly dependent on the circadian time of drug administration. Moreover, valsartan administration at bedtime as opposed to upon wakening results in improved diurnal/nocturnal ratio, a significant increase in the percentage of patients with controlled BP after treatment, and significant reductions in urinary albumin excretion and plasma fibrinogen. Chronotherapy provides a means of individualizing treatment of hypertension according to the circadian BP profile of each patient, and constitutes a new option to optimize BP control and reduce risk.
\end{abstract}

Keywords: valsartan, ambulatory blood pressure monitoring, dipper, nondipper, hypertension, chronotherapy, circadian rhythm

\section{Introduction}

Blood pressure (BP) and heart rate (HR) are characterized by predictable changes during the $24 \mathrm{~h}$, for the most part in synchrony with the rest-activity cycle (Lemmer 1992; Hermida et al 2002). This circadian variation in BP represents, on the one hand, the influence of internal factors such as ethnicity, gender, autonomic nervous system tone, vasoactive hormones, and hematologic and renal variables (Lemmer 1992; Sica and Wilson 2000). On the other hand, BP is affected by a variety of external factors including ambient temperature/humidity, physical activity, emotional state, alcohol or caffeine consumption, meal composition, and sleep/wake routine (Baumgart 1991; Portaluppi and Smolensky 2000; Hermida et al 2002).

Some specific features of the $24 \mathrm{~h} \mathrm{BP}$ pattern are linked to the progressive injury of target tissues and the triggering of cardiac and cerebrovascular events. In particular, many studies show the extent of the nocturnal BP decline is deterministic of cardiovascular injury and risk. The reduction of the assumed normal $10 \%$ to $20 \%$ sleep-time BP decline (nondipper pattern) is indeed associated with elevated risk of end-organ injury, particularly to the heart (left ventricular hypertrophy and myocardial infarct), brain (stroke), and kidney (albuminuria and progression to end-stage renal failure) (O'Brien et al 1988; Verdecchia et al 1994; Staessen et al 1999; Ohkubo et al 2002). O'Brien and colleagues (1988) reported that nondipper hypertensive subjects 
are significantly more likely to suffer a stroke than dippers. Verdecchia and colleagues (1994) also showed that, after an average follow-up period of 3.2 years, nondipper hypertensive patients experienced nearly 3 times as many adverse cardiovascular events as dippers. More recently, Staessen and colleagues (1999), summarizing results from the Syst-Eur trial where nitrendipine was dosed at bedtime, reported that non-dippers experienced a greater incidence of stroke and myocardial infarction than the group of persons who had a normal dipping pattern. Results from this trial also suggested that nighttime BP was the best predictor of one's risk to stroke and myocardial infarction. Moreover, Ingelsson and colleagues (2006) demonstrated that a nondipping BP pattern and increased nighttime diastolic BP (DBP) is a predictor of incident congestive heart failure in elderly men. These authors also addressed the issue of whether the loss of nocturnal BP reduction per se or an increased $24 \mathrm{~h}$ BP load causes organ damage. After inclusion of $24 \mathrm{~h}$ ambulatory systolic BP (SBP) and DBP as covariates in addition to antihypertensive treatment and established risk factors for congestive heart failure, nondipping and nighttime DBP remained significant predictors of congestive heart failure, indicating that the nondipping BP pattern per se is important.

The evaluation of the data from the Ohasama study indicated, after an average follow-up of 9.2 years, that a $5 \%$ decrease in the decline of nocturnal BP in hypertensive patients was associated with a $31 \%$ increased risk of cardiovascular mortality (Ohkubo et al 2002). What is even more relevant, dipper hypertensives had a relative hazard of cardiovascular mortality similar to that of nondipper normotensives (Ohkubo et al 2002). These results indicate that cardiovascular risk could be influenced not by BP elevation alone, but also by the magnitude of the circadian BP variability. In keeping with the results from the SystEur trial, a more recent evaluation of the Ohasama study has indicated, after 10.8 years of follow-up, that nighttime BP has a better prognostic value than daytime BP (Kikuya et al 2005). Accordingly, there is growing interest in how to tailor the treatment of hypertensive patients according to their circadian BP pattern (Ohkubo et al 2002; Hermida and Smolensky 2004).

Because the main steps in the mechanisms regulating BP are circadian-grade-dependent (Portaluppi and Smolensky 2000), it is not surprising that antihypertensive medications may display a circadian time-dependency in their pharmacokinetics and effects (Lemmer 2000; Hermida and Smolensky 2004). Chronotherapeutics has been defined as the purposeful timing of medications to proportion their serum and tissue concentrations in synchrony with known circadian rhythms in disease processes and symptoms as a means of enhancing beneficial outcomes and/or attenuating or averting adverse effects (Smolensky and Haus 2001). The chronotherapy of hypertension takes into account the epidemiology of the day-night BP pattern, plus potential administration-time determinants of the pharmacokinetics and dynamics of antihypertensive medications, as a means of enhancing beneficial outcomes and/or attenuating or averting adverse effects (Smolensky and Haus 2001). Specifically, it entails the attenuation of the accelerated morning rise of SBP and DBP along with the normalization of elevated daytime, nighttime and $24 \mathrm{~h}$ BP means, towards a dipper BP pattern associated with lower cardiovascular risk.

\section{Chronotherapy of antihypertensive medication: effects on the circadian BP pattern}

Appreciable ingestion-time differences in the kinetics (ie, chronokinetics) of BP-lowering and cardiac medications are well known (Lemmer and Portaluppi 1997; Smolensky 1997; Lemmer 2000). They result from circadian rhythms in gastric $\mathrm{pH}$ and emptying, gastrointestinal motility, biliary function and circulation, liver enzyme activity, and blood flow to the duodenum, kidney and other organs, among other factors (Labrecque and Beauchamp 2003). Clinically relevant dosing-time differences in the beneficial and adverse effects (termed chronodynamics) of BP-lowering medications are also known. They result from the chronokinetics of the medications as well as circadian rhythms in drug-free fraction, rate-limiting steps of key metabolic pathways, receptor number and conformation and/ or second messenger dynamics (Witte and Lemmer 2003). Differences in efficacy depending on the time of day of drug administration lead to differences in effects on the circadian pattern of BP and, in particular, on the nocturnal decline relative to the diurnal mean of BP (the diurnal/nocturnal $\mathrm{BP}$ ratio, an index of $\mathrm{BP}$ dipping).

\section{Chronotherapy with calcium channel blockers}

Several trials have investigated the differential effects of morning versus evening administration of calcium channel blockers (CCB) including amlodipine (Mengden et al 1992; Nold et al 1998; Qiu et al 2003), cilnidipine (Kitahara et al 
2004), diltiazem (Kohno et al 1997), isradipine (Fogari et al 1993; Portaluppi et al 1995), nifedipine (Greminger et al 1994; Hermida et al 2005a), nisoldipine (White et al 1999), and nitrendipine (Meilhac et al 1992; Umeda et al 1994). A sustained-release formulation of diltiazem was more effective on the $24 \mathrm{~h}$ mean BP when administered at night, although reducing the diurnal/nocturnal BP ratio towards a more nondipper profile (Kohno et al 1997). Dihydropyridine derivatives, differing in pharmacokinetics, seem to reduce BP homogeneously during day and night independently of dosing time. While peripheral edema seems to be more prevalent with dihydropyridine $\mathrm{CCB}$ as compared with other antihypertensive drugs, the incidence of this secondary effect might be reduced with evening as compared with morning dosing. The potential reduction of secondary effects with evening administration of $\mathrm{CCB}$ has already been taken into account in the design of previous trials. In the Syst-Eur trial mentioned above, where the objective was to investigate if active treatment (as compared with placebo) could reduce cardiovascular complications in elderly patients with isolated systolic hypertension, the studied drug (nitrendipine) was administered in the evening (Staessen et al 1999).

There are, however, some exceptions to the general conclusion that CCB have similar efficacy independently of dosing time. Umeda and colleagues (1994) reported that the effect of treatment with nitrendipine on the nocturnal mean of BP was significantly greater when the drug was administered in the evening, while also increasing the diurnal/nocturnal BP ratio towards a more dipper profile. Similar findings were reported by Portaluppi and colleagues (1995) who explored the relative advantage of evening versus morning once-a-day treatment with isradipine on the nondipping $24 \mathrm{~h}$ BP pattern in patients with chronic renal failure. The findings of this study demonstrated that in those nondipper chronic renal failure patients an evening, as compared to a morning, isradipine treatment schedule is more likely to effectively reduce the $24 \mathrm{~h}$ mean SBP and DBP and to restore the normal nocturnal dipping and circadian BP patterning.

\section{Chronotherapy with angiotensin- converting enzyme inhibitors}

Clinical studies demonstrated a different effect of the angiotensin-converting enzyme inhibitors (ACEI) benazepril (Palatini et al 1993), enalapril (Witte et al 1993), perindopril (Morgan et al 1997), quinapril (Palatini 1992), ramipril (Myburgh et al 1995), and trandolapril (Kuroda et al 2004) when dosed in the morning versus the evening. In all cases, evening administration of the drugs resulted in a higher effect on nocturnal BP and a significant modification of the circadian profile of BP. In a cross-over study on 20 hypertensive patients, Kohno and colleagues (2000) found no significant differences between the reduction in diurnal and nocturnal BP means after $10 \mathrm{mg}$ /day imidapril administered either in the morning or in the evening.

Similarly to most ACEI investigated so far, ramipril was more effective on the diurnal values of BP when administered in the morning and more effective on nocturnal BP when administered in the evening (Myburgh et al 1995). In the HOPE (Heart Outcomes Prevention Evaluation) study (Yusuf et al 2000) patients in the active treatment group received ramipril at bedtime. Results from a small substudy where patients were evaluated with ambulatory BP monitoring (ABPM) showed a marked BP reduction particularly during nighttime sleep (Svensson et al 2001), thereby reducing the prevalence of nondippers. The authors concluded that the effects on cardiovascular morbidity and mortality seen with ramipril in the HOPE study may relate to effects on BP patterns over the $24 \mathrm{~h}$ period (increase in the diurnal/nocturnal BP ratio) (Svensson et al 2001).

\section{Chronotherapy with $\alpha$-adrenoceptor antagonists}

Alpha-adrenoceptor blockade more effectively reduces peripheral resistance in the early morning hours than at other times of the day (Panza et al 1991). Indeed, a single nighttime dose of the $\alpha$-blocker doxazosin reduced both SBP and DBP throughout day and night, but its greatest effect was exerted early in the morning (Pickering et al 1994). Interestingly, the peak effect of doxazosin following nighttime dosing occurred later than predicted based upon the pharmacokinetic criteria (Pickering et al 1994), suggesting a circadian stage dependency in the doseresponse relationship, such as previously detected for nifedipine (Lemmer et al 1991), enalapril (Witte et al 1993), and propranolol (Langner and Lemmer 1988).

A recent study exploring the administration-timedependent effects of the doxazosin gastrointestinal therapeutic system (GITS) formulation (Hermida et al 2004) involved 91 subjects with stage 1 or 2 essential hypertension (Guidelines Committee 2003), randomly assigned to receive the daily dose of doxazosin GITS ( $4 \mathrm{mg} /$ day) either upon awakening or at bedtime for three months. Daily ingestion 
of doxazosin GITS upon awakening caused only a small and nonstatistically significant BP reduction, mainly because of an absence of drug effect on nocturnal B, thus significantly reducing the diurnal/nocturnal BP ratio towards a more nondipper profile. In contrast, daily ingestion of the medication at bedtime resulted in a statistically significant $\mathrm{BP}$ reduction. In summary, doxazosin GITS ingested daily upon awakening failed to provide full $24 \mathrm{~h}$ therapeutic coverage, while bedtime dosing significantly reduced BP throughout the entire $24 \mathrm{~h}$, whether used alone as a monotherapy or in combination with other antihypertensive medications (Hermida et al 2004).

In another recent trial, Kamoi and Ikarashi (2005) found that bedtime administration of doxazosin was safe and effective in reducing morning hypertension and albuminuria in patients with type-2 diabetes. On the other hand, the ALLHAT study (2000) indicated that treatment with doxazosin increased the risk of stroke and combined cardiovascular diseases and heart failure (Davis et al 2002). In this trial, doxazosin was taken once daily in the morning. Such administration may not be the best preference to adequately control high BP throughout the $24 \mathrm{~h}$, as previously documented (Pickering et al 1994; Hermida et al 2004).

\section{Chronotherapy with ß-adrenoceptor antagonists}

A summary of conventional studies (with drugs administered without time specification) (Stanton and O'Brien 1994) showed that $\beta$-blockers either have no effect or reduce the rhythmic pattern in BP towards a more nondipper profile. In general, there is a tendency for $\beta$-blockers to predominantly reduce diurnal BP and not to greatly affect nighttime values (Stanton and O'Brien 1994; Lemmer and Portaluppi 1997). In healthy subjects, a crossover study with propanolol showed a higher decrease in BP and HR during daytime hours than at night (Langner and Lemmer 1988). The higher impact of $\beta$-blockers on diurnal than on nocturnal BP correlates with the circadian rhythm in sympathetic tone, as indicated by the circadian rhythm in plasma noradrenaline (de Leeuw et al 1977).

In a recent study, we investigated the administration time-dependent antihypertensive efficacy of nebivolol in the presumably first chronotherapy trial with a $\beta$-blocker (Hermida et al 2005b). In this trial, patients with essential hypertension were randomly assigned to receive nebivolol monotherapy ( $5 \mathrm{mg} /$ day) either on awakening or at bedtime for two months. The diurnal/nocturnal BP ratio was significantly reduced towards a more nondipping pattern only after morning dosing, and was mainly unchanged after bedtime treatment. Results suggested that dosing time with nebivolol should be chosen at bedtime, without any loss in efficacy as compared with the usual morning administration, but avoiding the reduction in diurnal/nocturnal BP ratio that seems to be associated with higher cardiovascular risk.

\section{Chronotherapy with angiotensin II receptor blockers}

Angiotensin II receptor blockers (ARB) selectively and specifically antagonize the action of angiotensin II, a potent vasoconstrictor impacting $\mathrm{BP}$ regulation. ARBs are becoming increasingly popular because they are effective and well tolerated (Guidelines Committee 2003). A recent study (Hermida et al 2003a) used $48 \mathrm{~h} \mathrm{ABPM}$ to assess the antihypertensive efficacy of the ARB valsartan when ingested by stage 1 or 2 essential hypertension patients for three months, either in the morning after awakening from nighttime sleep or at bedtime. The highly significant BP reduction after treatment with $160 \mathrm{mg}$ /day valsartan was similar for both treatment times. Valsartan administration at bedtime as opposed to upon awakening, however, resulted in a highly significant average increase by $6 \%$ in the diurnal/ nocturnal $\mathrm{BP}$ ratio, corresponding to a $73 \%$ relative reduction from baseline in the number of nondipper patients (Hermida et al 2003a). The findings suggested the dosing time of valsartan could be chosen in relation to the dipper status of a given patient to improve therapeutic benefit and reduce cardiovascular risk. These results have been recently corroborated by two independent prospective chronotherapy trials, the first on elderly hypertensive patients (markedly characterized by a progressive reduction in diurnal/nocturnal BP ratio with aging) (Hermida et al 2005c), and the second on nondipper hypertensive patients (Hermida et al 2005d).

In the first of these trials, we studied 100 elderly patients with grade 1-2 essential hypertension randomly assigned to receive valsartan $(160 \mathrm{mg} /$ day $)$ either upon awakening or at bedtime. There was a highly significant $\mathrm{BP}$ reduction after three months of valsartan $(\mathrm{p}<0.001)$, slightly larger with bedtime than with morning dosing. The diurnal/ nocturnal BP ratio was unchanged in the group ingesting valsartan upon awakening, but significantly increased when valsartan was ingested at bedtime. Results from this trial suggest that in elderly hypertensive patients, characterized by a diminished nocturnal decline in BP, bedtime valsartan 
dosing is better than morning dosing since it improves efficacy during the nighttime sleep span, with the potential reduction in cardiovascular risk that has been associated with the normalization of the diurnal/nocturnal BP ratio.

In the second trial, we used a similar design to investigate the administration time-dependent effects on BP of the same dose of valsartan $(160 \mathrm{mg} /$ day $)$ in a selected population of nondipper hypertensive patients (Hermida et al 2005d). The significant BP reduction after three months of treatment was similar for both treatment times. The diurnal/nocturnal BP ratio was significantly increased only when valsartan was administered before bedtime, which resulted in $75 \%$ of the patients in this group reverting to dippers, a significant increase in the percentage of patients with controlled ambulatory BP after treatment, and a significant reduction in urinary albumin excretion (Hermida et al 2005d).

\section{Further evidence on the time- dependent antihypertensive efficacy of valsartan}

As indicated above, there is growing interest in how to treat nondipper hypertensive patients, due to their increasing cardiovascular risk and potential differing response to treatment as compared with patients with a normal dipping BP pattern. Some studies have indeed found differential effects of antihypertensive medications according to the dipping status of the patients (Ebata et al 1995; Kario and Shimada 1997; Kohno et al 1999, 2000; Kario et al 2000). However, efficacy studies of antihypertensive drugs in selected populations of nondipper patients are scarce. Accordingly, we have attempted to corroborate earlier conclusions (Hermida et al 2005d) evaluating the antihypertensive efficacy of valsartan monotherapy when dosed either in the morning after awakening from nighttime sleep or at bedtime for a three-month span in nondipper hypertensive patients.

\section{Methods}

\section{Subjects}

This prospective chronotherapy trial was conducted at the Hypertension and Vascular Risk Unit, Hospital Clínico Universitario, Santiago de Compostela, Spain. Shiftworkers, heavy drinkers (alcohol intake $>80 \mathrm{~g} / \mathrm{d}$ ), smokers ( $>20$ cigarettes/d), and heavy exercisers were excluded, as were individuals with either severe arterial (grade 3, that is BP $\geq 180 / 110 \mathrm{~mm} \mathrm{Hg}$ ) or secondary arterial hypertension and cardiovascular disorders, including angina, heart failure, stroke, nephropathy, and retinopathy or prior myocardial infarction or coronary revascularization as revealed by thorough clinical evaluation according to the standardized protocol at the Unit. Inclusion criteria required a diagnosis of grade 1 or 2 essential hypertension according to criteria of the recent European Society of Hypertension-European Society of Cardiology guidelines (Guidelines Committee 2003) based on conventional BP measurements (SBP between $140 \mathrm{~mm} \mathrm{Hg}$ and $179 \mathrm{~mm} \mathrm{Hg}$ or DBP between $90 \mathrm{~mm} \mathrm{Hg}$ and $109 \mathrm{~mm} \mathrm{Hg}$ ) and corroboration by ABPM at the time of recruitment. A positive diagnosis of hypertension based on ABPM in this trial required that either the $24 \mathrm{~h}$ mean SBP/DBP be above $130 / 80 \mathrm{~mm} \mathrm{Hg}$, the diurnal mean be above $135 / 85 \mathrm{~mm} \mathrm{Hg}$, or the nocturnal mean be above 120/70 mm Hg (O'Brien and Staessen 1995; O'Brien et al 2003; Pickering et al 2005). Moreover, inclusion criteria required all patents to be nondipper (here defined as $<10 \%$ decline in nocturnal mean compared with the diurnal mean of SBP using all data sampled by ABPM for 48 consecutive hours). With all these inclusion/exclusion criteria we identified 207 subjects who were accordingly randomized for the trial. Among these, 200 subjects (78 men and 122 women), $53.9 \pm 13.2$ years of age, completed the study.

After providing signed informed consent to participate in this prospective, randomized, open-label, blinded endpoint (PROBE), parallel-group chronotherapy trial, and after a 2- to 4-week wash-out period when required (78\% of the participants were previously never treated for hypertension and an additional $11 \%$ were untreated for at least 6 months), subjects were randomly assigned to receive single daily valsartan monotherapy $(160 \mathrm{mg}$ /day; the highest recommended and most widely used dose in Spain) either in the morning on awakening from nighttime sleep or at bedtime. Benefits of the PROBE design and its validity as compared with double-blind, placebo-controlled trials, in assessing antihypertensive efficacy based on blinded ABPM measurements have been previously documented (Smith et al 2003). The State Ethics Committee of Clinical Research approved the study.

Clinic BP measurements (6 per study visit after being seated for at least 5 minutes, on the same day just before starting ABPM) were always obtained by the same investigator with a validated automatic oscillometric device (HEM-737, Omron Health Care Inc., Vernon Hills, Illinois, USA) (Anwar et al 1998). Assignment of subjects to treatment groups was done by one member of the research team, according to the order of recruitment, following an allocation table constructed by a computerized random- 
number generator. The assignment of subjects to the respective treatment-time groups was blinded from the research team member conducting the clinic BP measurements and from those performing the statistical analysis of the data.

\section{ABPM assessment}

The SBP, DBP, and HR of each participant were automatically measured every 20 minutes from 07:00 to 23:00 and every 30 minutes during the night for 48 consecutive hours with a properly calibrated SpaceLabs 90207 device (SpaceLabs Inc., Issaquah, Washington, USA). Subjects were studied by ABPM under baseline conditions when subjects were free of medication (after washout in previously treated subjects), and again after 3 months of timed therapy. Participants were instructed to go about their usual activities with minimal restrictions but to follow a similar schedule during the two days of ABPM and to avoid daytime napping. No one was hospitalized during monitoring. ABPM always began between 10:00 and 12:00. BP series were not considered valid for analysis if more than $30 \%$ of the measurements were lacking or if they had missing data for $>2 \mathrm{~h}$ spans, or if they were collected from subjects while experiencing an irregular rest-activity schedule or a nighttime sleep span $<6$ h or $>12$ h during monitoring. Protocol-correct data series were collected from 200 subjects and therefore included in this efficacy stuty. Baseline BP profiles of 7 additional subjects ( 4 originally assigned to morning treatment and 3 to bedtime treatment) were eliminated because the patients failed to return for the second ABPM at the end of treatment.

\section{Actigraphy}

During $48 \mathrm{~h}$ ABPM each participant wore a Mini-MotionLogger actigraph (Ambulatory Monitoring Inc., Ardsley, New York, USA) on the dominant wrist to monitor physical activity every minute. This compact (about half the size of a wrist watch) device functions as an accelerometer. The internal clocks of the actigraph and the ABPM devices were synchronized through their respective interfaces by the same computer. The actigraphy data were used to determine the onset and offset times of diurnal activity and nocturnal sleep so as to accurately determine the diurnal and nocturnal BP means of each subject. The mean activity for the 5 minutes before each BP reading was then calculated for further statistical analysis on circadian variability of activity, according to previous studies on this area (Mansoor et al 2000; Hermida et al 2002a).

\section{Statistical methods}

Each individual's clock hour BP and HR values were first re-referenced from clock time to hours after awakening from nocturnal sleep, according to the information obtained from wrist actigraphy. This transformation avoided the introduction of bias due to differences among subjects in their sleep/activity routine (Hermida et al 2002a). BP and HR time series were then edited according to conventional criteria to remove measurement errors and outliers (Staessen et al 1991). Thus, readings with $\mathrm{SBP}>250 \mathrm{~mm} \mathrm{Hg}$ or $<70 \mathrm{~mm} \mathrm{Hg}$, DBP $>150 \mathrm{~mm} \mathrm{Hg}$ or $<40 \mathrm{~mm} \mathrm{Hg}$, and pulse pressure (PP, difference between SBP and DBP) $>150 \mathrm{~mm}$ $\mathrm{Hg}$ or $<20 \mathrm{~mm} \mathrm{Hg}$ were automatically discarded. For descriptive purposes, the circadian rhythm of BP, HR, and wrist activity before and after 3 months of intervention was objectively assessed by population multiple-component analysis (Fernández and Hermida 1998), a method applicable to nonsinusoidal shaped hybrid time series data (time series collected from a group of subjects) consisting of values distributed at equal or unequal intervals. The circadian rhythm parameters of MESOR (average value of the rhythmic function fitted to the data), overall amplitude (one-half the difference between the maximum and the minimum values of the best fitted curve), and orthophase (peak time, expressed as a lag from the time of awakening from nocturnal sleep) obtained for each group of patients before and after intervention were compared with a paired nonparametric test developed to assess differences in parameters derived from population multiple-components analysis (Fernández et al 2004). Hourly BP means obtained before and after intervention were compared by t-test corrected for multiple testing with the Holm procedure (Holm 1979). The daily (24h), diurnal, and nocturnal means of BP were further compared among treatment groups by ANOVA.

\section{Results}

\section{Efficacy with valsartan on awakening}

The circadian rhythm of SBP and DBP before and after treatment with valsartan on awakening is depicted in Figures 1 and 2 (left graphs). Morning valsartan treatment resulted in a statistically significant reduction in BP from baseline after 3 months of treatment $(13.0 \mathrm{~mm} \mathrm{Hg}$ and $8.1 \mathrm{~mm} \mathrm{Hg}$ reduction in the $24 \mathrm{~h}$ mean of SBP and DBP, respectively; $\mathrm{p}<0.001)$. After treatment, $66 \%$ of the patients had a controlled diurnal BP (below 135/85 mm Hg), but only $33 \%$ had a controlled nocturnal BP (below 120/70 mm Hg). There was also a significant reduction $(\mathrm{p}<0.001)$ of $4.8 \mathrm{~mm} \mathrm{Hg}$ in 
$1-1$ BEFORE TREATMENT

2 - - 2 AFTER TREATMENT

$* \mathrm{P}<0.005$ FROM $\mathrm{t}$-TEST ADJUSTED FOR MULTIPLE TESTING
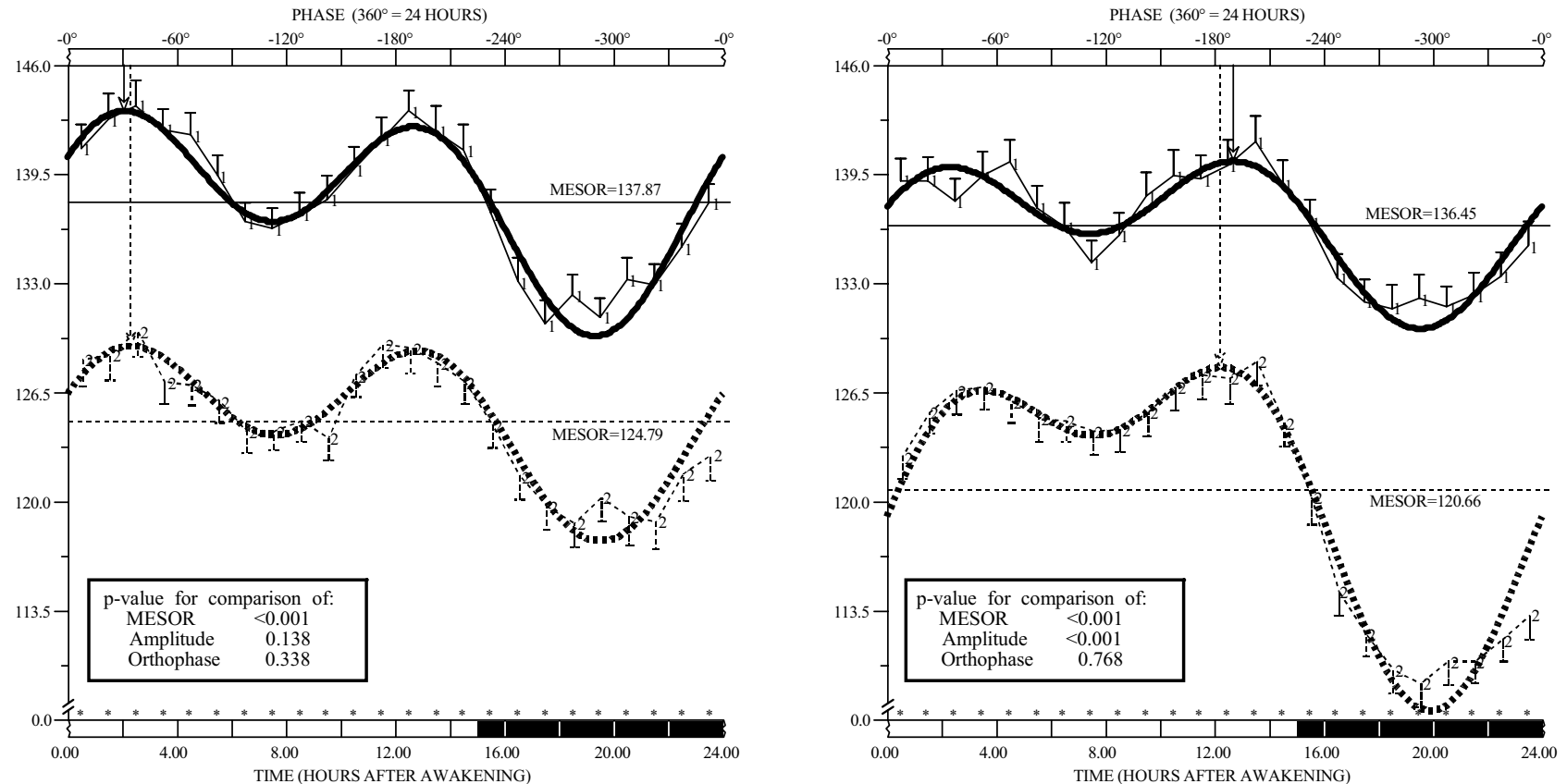

Figure I Circadian pattern of systolic blood pressure (SBP) before and after valsartan ( $160 \mathrm{mg} /$ day) administered on awakening (left) or at bedtime (right) in nondipper patients $(n=100)$ with grade I or 2 essential hypertension studied by $48 \mathrm{~h}$ ambulatory monitoring. Each graph shows hourly means and standard errors (SEs) of data collected before (continuous line) and after (dashed line) 3 months of treatment. Dark shading along the lower horizontal axis of the graphs represents average hours of nocturnal sleep across the patients. Nonsinusoidal-shaped curves represented around means and SEs correspond to the best-fitted waveform model determined by population multiple-component analysis. Arrows descending from upper horizontal axis point to the circadian orthophase (rhythm crest time).

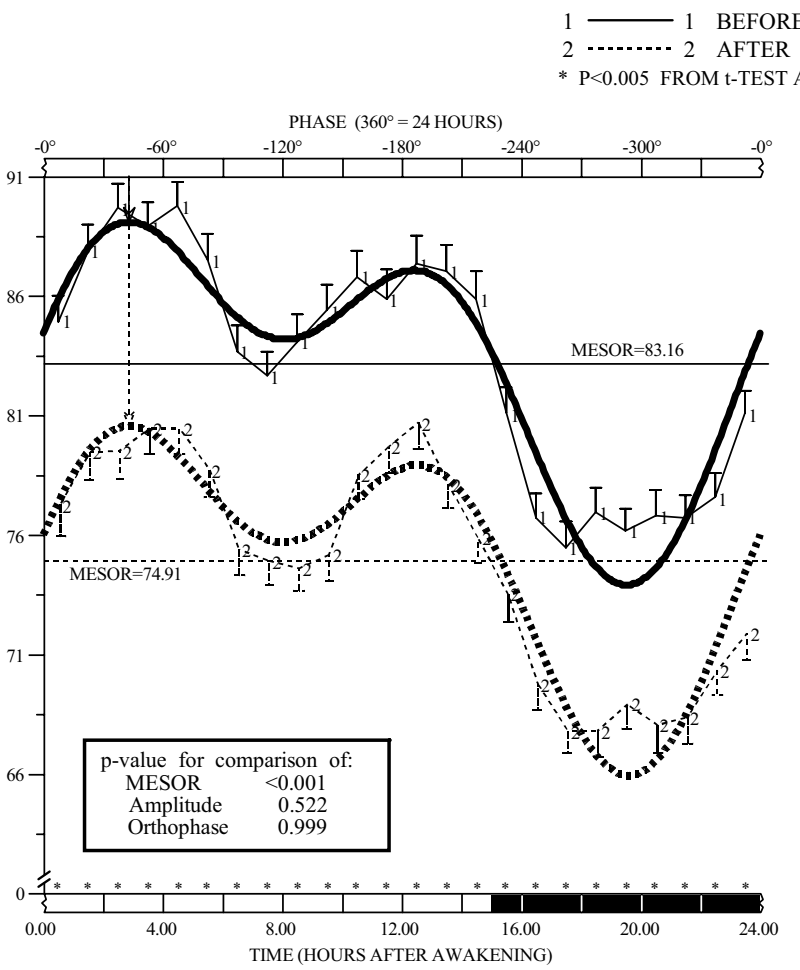

Figure 2 Circadian pattern of dystolic blood pressure (DBP) before and after valsartan ( $160 \mathrm{mg} /$ day) administered on awakening (left) or at bedtime (right) in nondipper patients $(\mathrm{n}=100)$ with grade I or 2 essential hypertension studied by $48 \mathrm{~h}$ ambulatory monitoring. Each graph shows hourly means and standard errors (SEs) of data collected before (continuous line) and after (dashed line) 3 months of treatment. Dark shading along the lower horizontal axis of the graphs represents average hours of nocturnal sleep across the patients. Nonsinusoidal-shaped curves represented around means and SEs correspond to the best-fitted waveform model determined by population multiple-component analysis. Arrows descending from upper horizontal axis point to the circadian orthophase (rhythm crest time). 
the $24 \mathrm{~h}$ mean of PP when valsartan was administered on awakening. Figures 1 and 2 (left panels) indicate a homogeneous BP reduction during the $24 \mathrm{~h}$ after treatment. These graphs further indicates that the mean reduction in $\mathrm{BP}$ at each clock time during the $24 \mathrm{~h}$ dosing interval was statistically significant ( $p$ always $<0.001$ after correcting for multiple testing), as designated by the asterisks above the lower horizontal axis. Despite the significant reduction in BP, there was no effect of valsartan on HR (decrease in the $24 \mathrm{~h}$ mean of 0.2 beats/minute, $p=0.782$ compared with baseline; not shown). The circadian pattern of physical activity monitored before and after 3 months of treatment also remained unchanged.

\section{Efficacy with valsartan at bedtime}

The graphs on the right of Figures 1 and 2 show the significant reduction compared with baseline of $15.2 \mathrm{~mm}$ $\mathrm{Hg}$ and $10.6 \mathrm{~mm} \mathrm{Hg}$ in the $24 \mathrm{~h}$ mean of SBP and DBP, respectively $(p<0.001)$ after 3 months of $160 \mathrm{mg} / \mathrm{d}$ valsartan taken at bedtime. After treatment, $76 \%$ of the patients had a controlled diurnal BP, and $59 \%$ had a controlled nocturnal BP. The $24 \mathrm{~h}$ mean of PP was also significantly reduced from baseline by $4.8 \mathrm{~mm} \mathrm{Hg}(\mathrm{p}<0.001)$. HR remained unchanged after 3 months of treatment (increase in the $24 \mathrm{~h}$ mean of 0.9 beats/minute, $p=0.122$ ). The circadian pattern of activity measured by wrist actigraphy was also similar before and after 3 months of therapy.

\section{Comparison between groups}

The comparison of results shown in Figures 1 and 2 revealed lack of statistically significant differences in ambulatory BP at baseline among the 2 treatment groups. After 3 months of timed treatment, the $24 \mathrm{~h}$ mean BP was also similar for both groups; accordingly, the treatment efficacy of valsartan on the $24 \mathrm{~h}$ of BP was comparable and independent of the time of its administration. The effect of medication on the nocturnal mean of BP was, however, significantly greater when valsartan was administered at bedtime. Figure 3 provides additional information on the comparison between the treatment groups of the changes in the diurnal, nocturnal, and $24 \mathrm{~h}$ mean BP values after 3 months of therapy. Figure 3 reveals the significant difference between the treatmentgroups in the effect of valsartan on the nocturnal mean of SBP and DBP $(\mathrm{p}<0.001)$.

When valsartan was taken on awakening, the mean reduction in the diurnal and nocturnal BP means was similar (Figure 3). However, when valsartan was taken at bedtime, the mean reduction in nocturnal BP was significantly greater than the mean reduction in diurnal BP. Accordingly, there was a statistically significant average increase $(\mathrm{p}<0.001)$ by $7.2 \%$ in the nocturnal decline in BP relative to the diurnal mean (an index of the BP dipping) only when valsartan was taken at bedtime (Figure 4), which resulted in $76 \%$ of the patients in this group reverted to a dipper BP pattern after 3 months of treatment.

\section{Discussion}

This prospective randomized trial focused on the potential benefits of chronotherapy in the specific treatment of nondipper hypertensive patients. Results, extending and complementing a previous trial on the general hypertensive population (Hermida et al 2003a), indicate that $160 \mathrm{mg} /$ day valsartan provide $24 \mathrm{~h}$ coverage when taken once daily either upon awakening or at bedtime. Valsartan, no matter the treatment time, also significantly reduced PP, a marker of cardiovascular risk. Valsartan dosing upon awakening and at bedtime similarly reduced BP during the $24 \mathrm{~h}$ (Figure 3 );
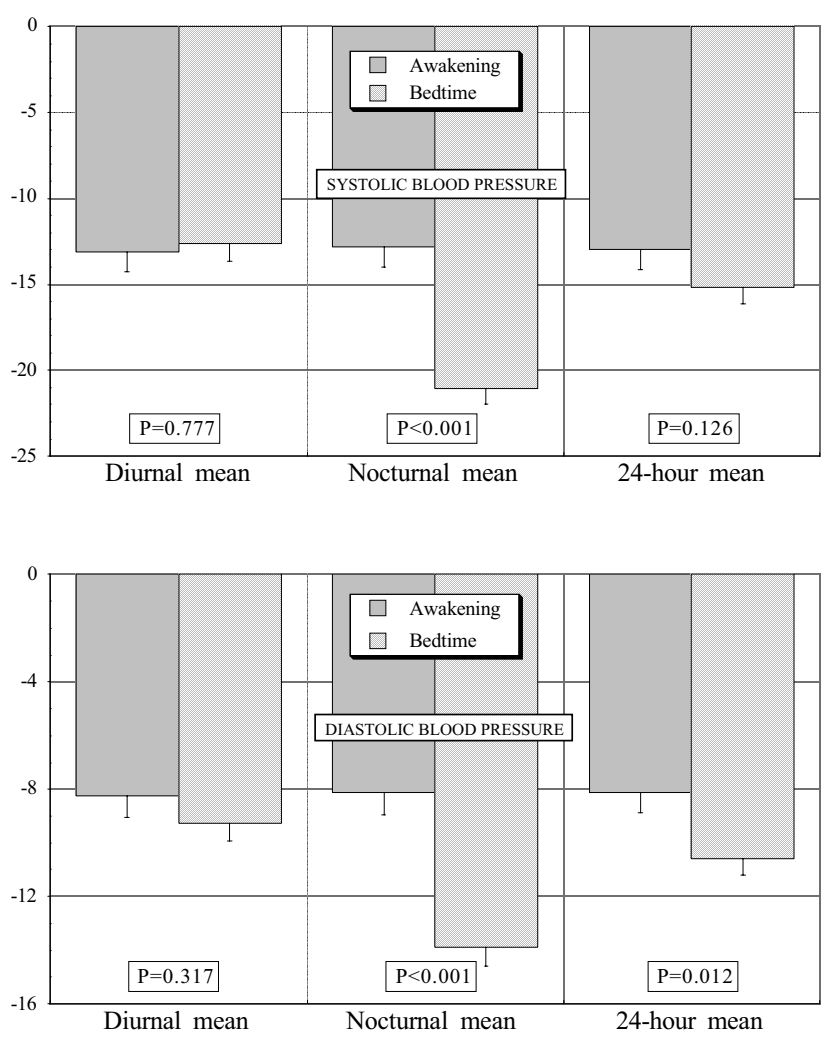

Figure 3 Effects on the diurnal, nocturnal, and $24 \mathrm{~h}$ mean of systolic blood pressure (SBP) (top) and dystolic blood pressure (DBP) (bottom) of valsartan $(160 \mathrm{mg} /$ day $)$ administered on awakening or at bedtime in nondipper patients with grade I or 2 essential hypertension studied by $48 \mathrm{~h}$ ambulatory monitoring before and after 3 months of treatment. Probability values are shown for comparison of effects between the 2 groups of subjects by ANOVA. 


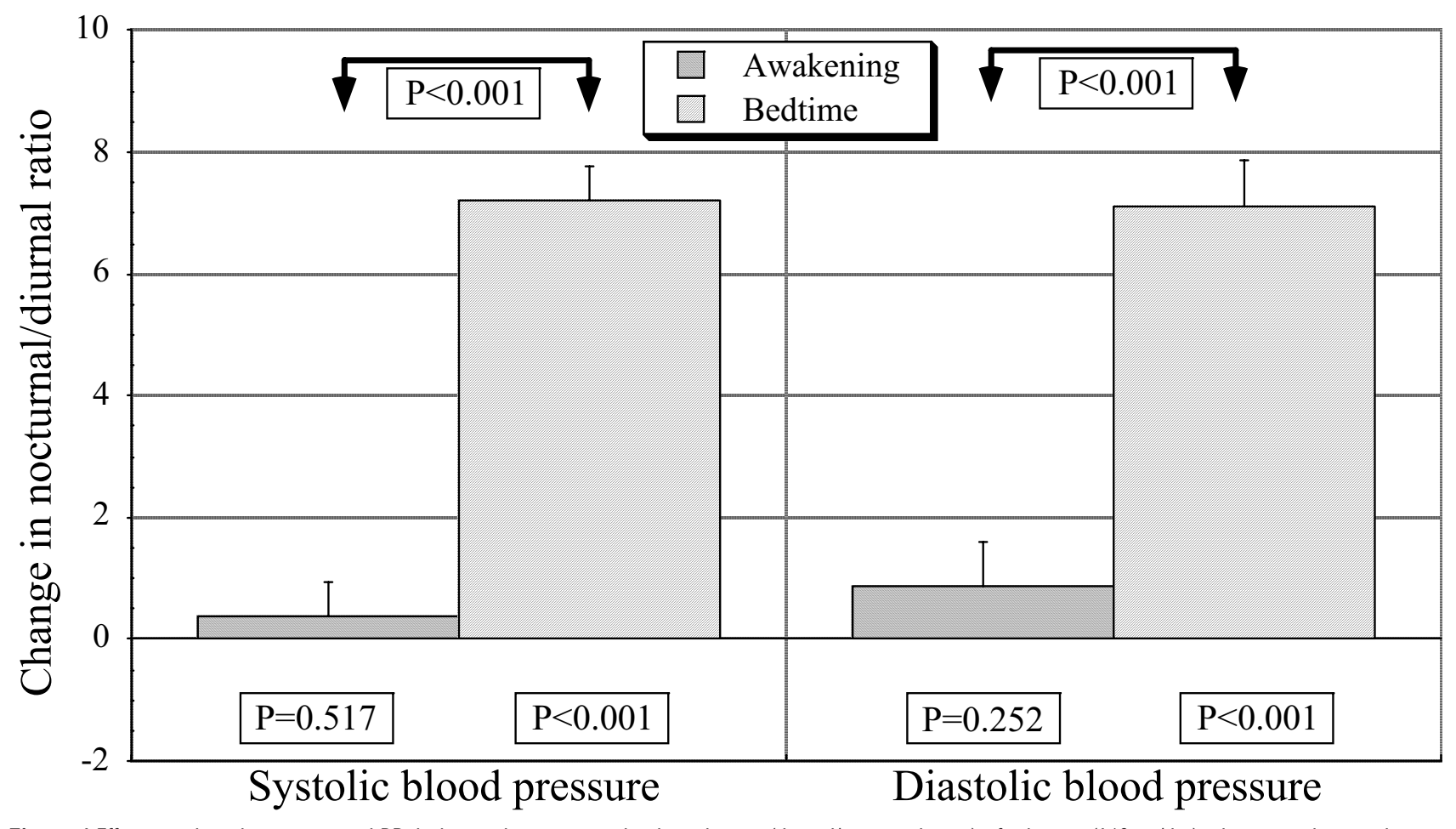

Figure 4 Effects on the relative nocturnal BP decline with respect to the diurnal mean (diurnal/nocturnal ratio) of valsartan ( $160 \mathrm{mg} /$ day) administered on awakening or at bedtime in nondipper patients with grade I or 2 essential hypertension studied by $48 \mathrm{~h}$ ambulatory monitoring before and after 3 months of treatment. $\mathrm{P}$ values on top are shown for comparison of effects among the 2 groups of patients by ANOVA. $P$ values on the bottom are shown for the change in diurnal/nocturnal ratio after treatment for every group and variable by paired t-test.

however, valsartan when administered at bedtime was especially efficient in reducing nocturnal $\mathrm{BP}$ and thus significantly increasing the diurnal/nocturnal ratio of BP (Figure 4). While in the general population (Hermida et al 2003a) the effects of treatment in nocturnal mean of BP were not statistically different between morning and bedtime dosing of valsartan, results in Figure 3 indicate that, in nondipper patients, the efficacy of valsartan at bedtime on the nocturnal mean of BP increases by $70 \%$ as compared with morning dosing. Other studies have also found differential effects of antihypertensive treatment between dipper and nondipper patients (Ebata et al 1995; Kario and Shimada 1997; Kohno et al 1999, 2000; Kario et al 2000).

Nocturnal hypertension, which is characterized by the loss or even reversal of the expected $10 \%-20 \%$ sleep-time BP decline, increases one's risk of cardiovascular and cerebrovascular events, nephrosclerosis, and progression to end-stage kidney failure in renal patients. Normalization of the circadian BP rhythm is considered to be an important clinical goal of pharmacotherapy because it may slow the advance of renal injury and avert end-stage renal failure (Timio et al 1993; Bianchi et al 1994). Nondipper BP patterning is more frequent in hypertension that is secondary to specific medical conditions, such as chronic renal failure, diabetes and autonomic nervous system dysfunction, than in uncomplicated primary hypertension (Hermida et al 2002). Results from a recent study, however, indicate a high $38 \%$ prevalence of nondipping among untreated patients with essential hypertension (Hermida et al 2002a). Most important, the percentage of nondipper patients significantly increase to $62 \%$ when they are evaluated by $48 \mathrm{~h} \mathrm{ABPM}$ under the effect of antihypertensive therapy. The percentage of patients who were receiving all their antihypertensive drugs in a single morning dose was significantly larger among nondippers (91\%) as compared with dippers (59\%), thus suggesting that nondipping among treated patients is partly due to the lack of $24 \mathrm{~h}$ therapeutic coverage of many antihypertensive drugs used in single morning dosing.

The definition of nocturnal hypertension on the basis of the dipper concept has been frequently criticized due to the inability to reproduce over time the classification of patients into dippers and nondippers (Mochizuki et al 1998). This may partly be due to the fact that most studies relied on $24 \mathrm{~h}$ monitoring. The advantages of $48 \mathrm{~h}$ sampling, instead of the most common $24 \mathrm{~h} \mathrm{ABPM}$, in terms of reproducibility of results and classification have been documented previously (Mochizuki et al 1998; Hermida et al 2002a, 2002c). Uncertainty in the classification of a patient as 
dipper may be reduced from $42 \%$ to just $6 \%$ by expanding monitoring from $24 \mathrm{~h}$ to $48 \mathrm{~h}$ (Hermida et al 2002b), as the proper estimation of mean BP values is more dependent on duration of monitoring than on sampling rate (Hermida and Ayala 2003).

International guidelines recommend the use of longacting, once-daily medications that provide $24 \mathrm{~h}$ efficacy; they improve adherence to therapy and minimize BP variability with with smoother and more consistent $\mathrm{BP}$ control (Chobanian et al 2003; Guidelines Committee 2003). Most antihypertensive medication, including valsartan, has been approved to be used once-daily, without any specification of treatment-time. Use of a medication with a high homogeneous efficacy along the $24 \mathrm{~h}$, such as amlodipine or valsartan administered on awakening, is unlikely to affect the circadian profile of BP and may be the best choice for the treatment of dipper hypertensive patients. This therapeutic scheme, however, may not be appropriate for managing nondippers, since it is important to avoid nocturnal hypertension (Staessen et al 1999; Kikuya et al 2005). The available scientific evidence suggests nondippers may benefit from evening dosing of certain BP-lowering medications to reduce abnormally high BP and to convert the disturbed $24 \mathrm{~h}$ BP profile to normal dipper pattern of lower cardiovascular risk. However, because the effects of BP medications can be circadian stage dependent, the specific administration-time-dependent dose-response curve of the drug must be determined and taken into consideration to treat hypertensive patients effectively. The most recent studies on the chronotherapy of hypertension summarized here confirm the effects of antihypertensive medications can vary, sometimes dramatically, as a function of their circadian time of dosing.

The potential reduction in cardiovascular risk associated with the normalization of the circadian variability of BP (converting a nondipper to dipper BP pattern) is still a matter of debate. Apart from the Syst-Eur trial where nitrendipine was administered at bedtime, results from the HOPE substudy where patients were evaluated by ABPM indicated a significant BP reduction mainly during hours of nighttime sleep (Svensson et al 2001). The authors suggested that the beneficial effects on cardiovascular morbidity and mortality in the HOPE study may be related to the $8 \%$ increase in the diurnal/nocturnal ratio of BP seen after ramipril was administered at bedtime. A recent study with another ACEI, temocapril, evaluated its time-dependent effect on the mortality of stroke-prone spontaneous hypertensive rats (Nozawa et al 2006). Temocapril prolonged the survival rate of the animals, with a maximum effect after dosing at the early resting period and a minimal effect after dosing at the early active period. These results are relevant inasmuch as, similarly to other ACEI, morning dosing with temocapril significantly reduces diurnal $\mathrm{BP}$ to a greater extent than nocturnal BP and thus decreases the diurnal/nocturnal BP ratio towards a more nondipper profile (Eguchi et al 2002).

Another relevant study where the nondipper BP profile in patients with chronic renal failure was normalized after evening but not after morning four-week dosing of isradipine (Portaluppi et al 1995) did not conduct follow-up to evaluate potential changes in cardiovascular risk, mainly due to the short period of active treatment. On the other hand, recent results have demonstrated that urinary albumin excretion was significantly reduced after bedtime, but not morning, treatment with valsartan (Hermida et al 2005e). This reduction was independent of the $24 \mathrm{~h}$ BP decrease after treatment, but highly correlated with the decrease in the nocturnal mean of BP and, mainly, with the increase in diurnal/nocturnal BP ratio associated with bedtime administration of valsartan. These results are of clinical importance, as there is growing evidence that reduction of urinary albumin excretion provides renal protection and reduces cardiovascular risk (Ruggenenti et al 2001).

Apart from this beneficial effect of chronotherapy on albumin, plasma fibrinogen has also been shown to be significantly reduced after bedtime treatment with valsartan in direct correlation with the increasing diurnal/nocturnal BP ratio resulting from the conversion of nondippers into dippers (Hermida et al 2005c, 2005e). Clinical trials and epidemiological observations have indicated that elevated plasma fibrinogen levels are strongly correlated with an increased frequency of vascular events, thus recognizing fibrinogen as a significant parameter for assessing the potential risk of acute myocardial infarction and stroke (Wilhelmsen et al 1984). Previous results have already established that plasma fibrinogen is significantly elevated in nondipper as compared with dipper hypertensives (Hermida et al 2003b). The reduction in fibrinogen in relation to the normalization of the BP profile, ie, conversion from nondipper to a dipper pattern, seems to provide further evidence of the potential reduction in cardiovascular risk associated with the normalization of the circadian BP pattern in hypertensive patients with altered nocturnal BP.

Common to all previous trials demonstrating the increased cardiovascular risk in non-dippers ( $\mathrm{O}^{\prime}$ Brien et al 1988; Verdecchia et al 1994; Staessen et al 1999; Ohkubo et al 2002) is that the prognostic significance of ABPM has 
relied on a single baseline profile from each participant, without accounting for possible changes in the BP pattern, mainly associated with antihypertensive therapy and aging during follow-up. Along these lines, the MAPEC study (Monitorización Ambulatoria de Presión arterial y Eventos Cardiovasculares) was designed to investigate whether normalizing the circadian BP profile towards a more dipper pattern by chronotherapy strategies reduces cardiovascular risk (Hermida et al 2006). This prospective study investigates over 2600 subjects from the health registry of Santiago de Compostela (Northwest Spain). At inclusion, BP was measured for 48 consecutive hours at 20-minute intervals from 07:00 to 23:00 and at 30-minute intervals at night. Physical activity was simultaneously monitored every minute by wrist actigraphy. Blood samples and $24 \mathrm{~h}$ urine collection were obtained the same day before starting ABPM. The same evaluation procedure is scheduled yearly (or more frequently, every three months, if treatment adjustment is required). Based on the baseline ABPM and after a median time of follow-up of 3.2 years, cardiovascular morbidity was similar for extreme-dippers (diurnal/nocturnal BP ratio $>20 \%$; 1.23 events per 100 patient-years) and dippers (1.14), but significantly higher for nondippers (2.81) and mainly for risers (patients with nocturnal BP mean above diurnal mean; 8.70 events per 100 patient-years). When cardiovascular morbidity was analyzed on the basis of the last ABPM profile closest to the cardiovascular event (or the last available evaluation for event-free patients), morbidity was found to be reduced in extreme-dippers $(0.38)$ and dippers (0.89), but increased in nondippers (3.23) and risers (10.70). All cases but one of cardiovascular death were associated with a riser or nondipper pattern prior to the event (Hermida et al 2006). Results from this ongoing prospective study indicate the probability of event-free survival is strongly correlated with the diurnal/nocturnal BP ratio. Most important, results suggest that increasing this ratio towards a more dipper pattern decreases cardiovascular risk, while decreasing the diurnal/nocturnal BP ratio is associated with increased morbidity and mortality. In the light of all these findings, evaluation of the potential decrease in cardiovascular risk from the proper modeling of the circadian BP profile by the timed administration of antihypertensive medication, beyond reduction of BP levels, deserves further prospective investigation.

\section{Acknowledgments}

This research was supported in part by grants from Xunta de Galicia (PGIDIT03-PXIB-32201PR); Hospital Clínico
Universitario de Santiago; and Vicerrectorado de Investigación, University of Vigo.

\section{References}

Anwar YA, Giacco S, McCabe EJ, et al. 1998. Evaluation of the efficacy of the Omron HEM-737 Intellisense device for use on adults according to the recommendations of the Association for the Advancement of Medical Instrumentation. Blood Press Monit, 3:261-5.

Baumgart P. 1991. Circadian rhythm of blood pressure: Internal and external triggers. Chronobiol Int, 8:444-50.

Bianchi S, Bigazzi R, Baldari G, et al. 1994. Diurnal variations of blood pressure and microalbuminuria in essential hypertension. $A m \mathrm{~J}$ Hypertens, 7:23-9.

Chobanian AV, Bakris GL, Black HR, et al. 2003. The seventh report of the Joint National Committee on Prevention, Detection, Evaluation, and Treatment of High Blood Pressure. JAMA, 289:2560-71.

Davis BR, Cutler JA, Furberg CD, et al. 2002. Relationship of antihypertensive treatment regimens and change in blood pressure to risk for heart failure in hypertensive patients randomly assigned to doxazosin or chlorthalidone: Further analyses from the antihypertensive and lipid-lowering treatment to prevent heart attack trial. Ann Intern Med, 137(5 part 1):313-20.

de Leeuw PW, Falke HE, Kho TL, et al. 1977. Effects of beta-agrenergic blockade on diurnal variability of blood pressure and plasma noradrenaline levels. Acta Med Scand, 202:389-92.

Ebata H, Hojo Y, Ikeda U, et al. 1995. Differential effects of an alpha 1blocker (doxazosin) on diurnal blood pressure variation in dipper and non-dipper type hypertension. Hypertens Res, 18:125-30.

Eguchi K, Kario K, Shimada K. 2002. Differential effects of a long-acting angiotensin converting enzyme inhibitor (temocapril) and a longacting calcium antagonist (amlodipine) on ventricular ectopic beats in older hypertensive patients. Hypertens Res, 25:329-33.

Fernández JR, Hermida RC. 1998. Inferential statistical method for analysis of nonsinusoidal hybrid time series with unequidistant observations. Chronobiol Int, 15:191-204.

Fernández JR, Mojón A, Hermida RC. 2004. Comparison of parameters from rhythmometric models with multiple components on hybrid data. Chronobiol Int, 21:469-84.

Fogari R, Malacco E, Tettamanti F, et al. 1993. Evening vs. morning isradipine sustained release in essential hypertension: a double-blind study with $24 \mathrm{~h}$ ambulatory monitoring. Br J Clin Pharmacol, 35:514.

Greminger P, Suter PM, Holm D, et al. 1994. Morning versus evening administration of nifedipine gastrointestinal therapeutic system in the management of essential hypertension. Clin Invest, 72:864-9.

Guidelines Committee. 2003. European Society of Hypertension European Society of Cardiology guiudelines for the management of arterial hypertension. J Hypertens, 21:1011-53.

Hermida RC, Smolensky MH. 2004. Chronotherapy of hypertension. Curr Opin Nephrol Hypertens, 13:501-5.

Hermida RC, Ayala DE, Fernández JR, et al. 2002. Modeling the circadian variability of ambulatorily monitored blood pressure by multiplecomponent analysis. Chronobiol Int, 19:461-81.

Hermida RC, Ayala DE. 2003. Sampling requirements for ambulatory blood pressure monitoring in the diagnosis of hypertension in pregnancy. Hypertension, 42:619-24.

Hermida RC, Calvo C, Ayala DE, et al. 2002a. Relationship between physical activity and blood pressure in dipper and nondipper hypertensive patients. $J$ Hypertens, 20:1097-104.

Hermida RC, Calvo C, Ayala DE, et al. 2002b. Evaluation of the extent and duration of the "ABPM effect" in hypertensive patients. $J \mathrm{Am}$ Coll Cardiol, 40:710-17.

Hermida RC, Calvo C, Ayala DE, et al. 2003a. Administration timedependent effects of valsartan on ambulatory blood pressure in hypertensive subjects. Hypertension, 42:283-90. 
Hermida RC, Calvo C, Ayala DE, et al. 2003b. Seasonal variation of fibrinogen in dipper and nondipper hypertensive patients. Circulation, 108:1101-6.

Hermida RC, Calvo C, Ayala DE, et al. 2004. Administration-timedependent effects of doxazosin GITS on ambulatory blood pressure of hypertensive subjects. Chronobiol Int, 21:277-96.

Hermida RC, Calvo C, Ayala DE, et al. 2005a. Administration timedependent effects of nifedipine GITS on ambulatory blood pressure in patients with essential hypertension. Am J Hypertens, 18(5 part 2):63A.

Hermida RC, Calvo C, Ayala DE, et al. 2005b. Administration timedependent effects of nebivolol on ambulatory blood pressure in patients with essential hypertension. Am J Hypertens, 18(5 part 2):63A.

Hermida RC, Calvo C, Ayala DE, et al. 2005c. Administration timedependent effects of valsartan on ambulatory blood pressure in elderly hypertensive subjects. Chronobiol Int, 22:755-76.

Hermida RC, Calvo C, Ayala DE, et al. 2005d. Treatment of non-dipper hypertension with bedtime administration of valsartan. J Hypertens, 23:1913-22.

Hermida RC, Calvo C, Ayala DE, et al. 2005e. Decrease in urinary albumin excretion associated to the normalization of nocturnal blood pressure in hypertensive subjects. Hypertension, 46:960-8.

Hermida RC, Calvo C, Ayala DE, et al. 2006. Prediction of cardiovascular events by ambulatory blood pressure and effects of Chronotheraoy: The MAPEC study. J Hypertens, 24(suppl 4):S180.

Holm S. 1979. A simple sequentially rejective multiple test procedure. Scand J Stat, 6:65-70.

Ingelsson E, Bjorklund-Bodegard K, Lind L, et al. 2006. Diurnal blood pressure pattern and risk of congestive heart failure. JAMA, 295:285966.

Kamoi K, Ikarashi T. 2005. The bedtime administration of doxazosin controls morning hypertension and albuminuria in patients with type2 diabetes: Evaluation using home-based blood pressure measurements. Clin Exp Hypertens, 4:369-76.

Kario K, Schwartz JE, Pickering TG. 2000. Changes of nortunal blood pressure dipping status in hypertensives by nighttime dosing of aadrenergic blocker, doxazosin. Results from the HALT study. Hypertension, 35:787-94.

Kario K, Shimada K. 1997. Differential effects of amlodipine on ambulatory blood pressure in elderly hypertensive patients with different nocturnal reductions in blood pressure. Am J Hypertens, 10:261-8.

Kikuya M, Ohkubo T, Asayama K, et al. 2005. Ambulatory blood pressure and 10-year risk of cardiovascular and noncardiovascular mortality. The Ohasama Study. Hypertension, 45:240-5.

Kitahara Y, Saito F, Akao M, et al. 2004. Effect of morning and bedtime dosing with cilnidipine on blood pressure, heart rate, and sympathetic nervous activity in essential hypertensive patients. $J$ Cardiovasc Pharmacol, 43:68-73.

Kohno I, Ijiri H, Takusagawa M, et al. 1999. Chronotherapy of dipper and non-dipper type hypertension with bunazisin-retard: Morning versus evening administration. Am J Hypertens, 12(4 Part 2):156A.

Kohno I, Ijiri H, Takusagawa M, et al. 2000. Effect of imidapril in dipper and nondipper hypertensive patients: comparison between morning and evening administration. Chronobiol Int, 17:209-19.

Kohno I, Iwasaki H, Okutani M, et al. 1997. Administration-timedependent effects of diltiazem on the 24-hour blood pressure profile of essential hypertension patients. Chronobiol Int, 14:71-84.

Kuroda T, Kario K, Hoshide S, et al. 2004. Effects of bedtime vs. morning administration of the long-acting lipophilic angiotensin-converting enzyme inhibitor trandolapril on morning blood pressure in hypertensive patients. Hypertens Res, 27:15-20.

Labrecque G, Beauchamp D. 2003. Rhythms and pharmacokinetics. In: Redfern P (ed). Chronotherapeutics. London, England: Pharmaceutical Pr, pp 75-110.
Langner B, Lemmer B. 1988. Circadian changes in the pharmacokinetics and cardiovascular effects of oral propranolol in healthy subjects. Eur $J$ Clin Pharmacol, 33:619-24.

Lemmer B, Nold G, Behne S, et al. 1991. Chronopharmacokinetics and cardiovascular effects of nifedipine. Chronobiol Int, 8:485-94.

Lemmer B, Portaluppi F. 1997. Chronopharmacology of cardiovascular diseases. In: Redfern P, Lemmer B (eds). Handbook of Experimental Pharmacology, Vol 125, Physiology and Pharmacology of Biological Rhythms. Heidelberg, Germany: Springer Verlag, pp 251-97.

Lemmer B. 1992. Cardiovascular chronobiology and chronopharmacology. In: Touitou Y, Haus E, (eds). Biologic Rhythms in Clinical and Laboratory Medicine. Berlin, Germany: Springer-Verlag, pp 418-27.

Lemmer B. 2000. Cardiovascular chronobiology and chronopharmacology. Importance of timing of dosing. In: White WB (ed). Blood Pressure Monitoring in Cardiovascular Medicine and Therapeutics. Totowa, NJ: Humana Pr, pp 255-271.

Mansoor GA, White WB, McCabe EJ, et al. 2000. The relationship of electronically monitored physical activity to blood pressure, heart rate, and the circadian blood pressure profile. Am J Hypertens, 13:262-7.

Meilhac B, Mallion JM, Carre A, et al. 1992. Study of the influence of the time of administration on the antihypertensive effect and nitrendipine tolerance in mild to moderate essential hypertensive patients. Value of ambulatory recording of blood pressure on 24 hours. Therapie, 47:205-10.

Mengden T, Binswanger B, Gruene S. 1992. Dynamics of drug compliance and 24-hour blood pressure control of once daily morning vs evening amlodipine. J Hypertens, 10(Suppl 4):S136.

Mochizuki Y, Okutani M, Donfeng Y, et al. 1998. Limited reproducibility of circadian variation in blood pressure dippers and nondippers. Am J Hypertens, 11:403-9.

Morgan T, Anderson A, Jones E. 1997. The effect on $24 \mathrm{~h}$ blood pressure control of an angiotensin converting enzyme inhibitor (perindopril) administered in the morning or at night. $J$ Hypertens, 15:205-11.

Myburgh DP, Verho M, Botes JH, et al. 1995. 24-Hour pressure control with ramipril: comparison of once-daily morning and evening administration. Curr Ther Res, 56:1298-306

Nold G, Strobel G, Lemmer B. 1998. Morning vs evening amlodipine treatment: effect on circadian blood pressure profile in essential hypertensive patients. Blood Press Monit, 3:17-25.

Nozawa M, Sugimoto K, Ohmori M, et al. 2006. Dosing time-dependent effect of temocapril on the mortality of stroke-prone spontaneously hypertensive rats. J Pharmacol Exp Ther, 316:176-81.

O'Brien A, Asmar R, Beilin L, et al. 2003. European Society of Hypertension recommendations for conventional, ambulatory and home blood pressure measurement. J Hypertens, 21:821-48.

O’Brien E, Sheridan J, O’Malley K. 1988. Dippers and non-dippers. Lancet, 13:397.

O'Brien E, Staessen J. 1995. Normotension and hypertension as defined by 24-hour ambulatory blood pressure monitoring. Blood Press, 4:26682.

Ohkubo T, Hozawa A, Yamaguchi J, et al. 2002. Prognostic significance of the nocturnal decline in blood pressure in individuals with and without high 24-h blood pressure: the Ohasama study. J Hypertens, 20:2183-9.

Palatini P, Mos L, Motolese M, et al. 1993. Effect of evening versus morning benazepril on 24-hour blood pressure: a comparative study with continuous intraarterial monitoring. Int J Clin Pharmacol Ther Toxicol, 31:295-300.

Palatini P, Racioppa A, Raule G, et al. 1992. Effect of timing of administration on the plasma ACE inhibitory activity and the antihypertensive effect of quinapril. Clin Pharmacol Ther, 52:37883.

Palatini P. 1992. Can an angiotensin-converting enzyme inhibitor with a short half-life effectively lower blood pressure for 24 hours? Am Heart $J, 123: 1421-5$. 
Panza JA, Epstein SE, Quyyumi AA. 1991. Circadian variation in vascular tone and its relation to alpha-sympathetic vasoconstrictor activity. $N$ Engl J Med, 325:986-90.

Pickering TG, Hall JE, Appel LJ, et al. 2005. Recommendations for blood pressure measurement in humans and experimental animals. Part 1: Blood pressure measurement in humans. Hypertension, 45:14261.

Pickering TG, Levenstein M, Walmsley P; for the Hypertension and Lipid Trial Study Group. 1994. Night-time dosing of doxazosin has peak effect on morning ambulatory blood pressure. Results of the HALT study. Am J Hypertens, 7:844-7.

Portaluppi F, Smolensky MH. 2000. Circadian rhythm and environmental determinants of blood pressure regulation in normal and hypertensive conditions. In: White WB (ed). Blood Pressure Monitoring in Cardiovascular Medicine and Therapeutics. Totowa, NJ: Humana Pr, pp 79-118.

Portaluppi F, Vergnani L, Manfredini R, et al. 1995. Time-dependent effect of isradipine on the nocturnal hypertension in chronic renal failure. Am J Hypertens, 8:719-26.

Qiu YG, Chen JZ, Zhu JH, et al. 2003. Differential effects of morning or evening dosing of amlodipine on circadian blood pressure and heart rate. Cardiovasc Drugs Ther, 17:335-41.

Ruggenenti P, Schieppati A, Remuzzi G. 2001. Progression, remission, regression of chronic renal disease. Lancet, 357:1601-8.

Sica DA, Wilson DK. 2000. Sodium, potassium, the sympathetic nervous system, and the renin-angiotensin system: Impact on the circadian variability in blood pressure. In: White WB (ed). Blood Pressure Monitoring in Cardiovascular Medicine and Therapeutics. Totoya, NJ: Humana Pr, pp 171-90.

Smith DH, Neutel JM, Lacourciere Y, et al. 2003. Prospective, randomized, open-label, blinded-endpoint (PROBE) designed trials yield the same results as double-blind, placebo-controlled trials with respect to ABPM measurements. J Hypertens, 21:1291-8.

Smolensky MH, Haus E. 2001. Circadian rhythms in clinical medicine with applications to hypertension. Am J Hypertens, 14:280S-90S.

Smolensky MH. 1997. Chronobiology and chronotherapeutics: Applications to cardiovascular medicine. In: Deedwania PC (ed). Circadian Rhythms of Cardiovascular Disorders. Armonk, N.Y.: Futura Publishing Co., Inc, pp 173-206.
Staessen J, Fagard R, Lijnen P, et al. 1991. Ambulatory blood pressure monitoring in clinical trials. J Hypertens, 9(Suppl 1):s13-19.

Staessen JA, Thijs L, Fagard R, et al. 1999. Predicting cardiovascular risk using conventional vs ambulatory blood pressure in older patients with systolic hypertension. JAMA, 282:539-546.

Stanton A, O'Brien E. 1994. Auswirkungen der Therapie auf das zirkadiane. Blutdruckprofil Kar-dio, 3:1-8.

Svensson P, de Faire U, Sleight P, et al. 2001. Comparative effects of ramipril on ambulatory and office blood pressures. A HOPE substudy. Hypertension, 38:e28-32.

The ALLHAT Collaborative Research Group. 2000. Major cardiovascular events in Hypertensive patients randomized to doxazosin vs. chlorthalidone: The antihypertensive and lipid-lowering treatment to prevent heart attack trial (ALLHAT). JAMA, 283:1967-75.

Timio M, Lolli S, Verdura C, et al. 1993. Circadian blood pressure changes in patients with chronic renal insufficiency: a prospective study. Ren Fail, 15:231-7.

Umeda T, Naomi S, Iwaoka T, et al. 1994. Timing for administration of an antihypertensive drug in the treatment of essential hypertension. Hypertension, 23(Suppl 1):I211-14.

Verdecchia P, Porcellati C, Schillaci G, et al. 1994. Ambulatory blood pressure: an independent predictor of prognosis in essential hypertension. Hypertension, 24:793-801.

White WB, Mansoor GA, Pickering TG, et al. 1999. Differential effects of morning and evening dosing of nisoldipine ER on circadian blood pressure and heart rate. Am J Hypertens, 12:806-14.

Wilhelmsen L, Svardsudd K, Korsan-Bengtsen K, et al. 1984. Fibrinogen as a risk factor for stroke and myocardial infarction. $N$ Engl $J$ Med, 311:501-5.

Witte K, Lemmer B. 2003. Rhythms and pharmacodynamics. In: Redfern P (ed). Chronotherapeutics. London, England: Pharmaceutical Pr, pp 111-26.

Witte K, Weisser K, Neubeck M, et al. 1993. Cardiovascular effects, pharmacokinetics and converting enzyme inhibition of enalapril after morning versus evening administration. Clin Pharmacol Ther. 54:17786.

Yusuf S, Sleight P, Pogue J, et al. 2000. Effects of an angiotensinconverting-enzyme inhibitor, ramipril, on cardiovascular events in high-risk patients: the Heart Outcomes Prevention Evaluation Study Investigators. $N$ Engl J Med, 342:145-53. 
